\title{
Heidelberg, II Physikalisches Institut
}

\begin{tabular}{|c|c|c|c|c|c|}
\hline $\begin{array}{l}\text { Laboratory } \\
\text { number }\end{array}$ & $\begin{array}{l}\text { Published } \\
\text { reference }\end{array}$ & $\begin{array}{l}\text { Original date or } \\
\quad \text { other value }\end{array}$ & $\begin{array}{l}\text { Corrected date } \\
\text { or other value }\end{array}$ & $\partial \mathrm{C}^{14}, \% 0$ & $\begin{array}{c}\text { A.D./ } \\
\text { B.C. date }\end{array}$ \\
\hline H-1-8 & $30: 196$ & $11,900 \pm 500$ & & & 9950 B.C. \\
\hline$-1-48$ & $30: 196$ & $11,800 \pm 300$ & & & 9850 B.C. \\
\hline$-8-7-$ & $30: 197$ & $1245 \pm 130$ & & & A.D. 705 \\
\hline$-11-29$ & $30: 195$ & $+1.51 \pm 0.73$ & $+18.1 \pm 7.3 \mathrm{a}$ & & \\
\hline$-11-31$ & $30: 195$ & $-0.82 \pm 0.47$ & $-5.2 \pm 4.7$ a & & \\
\hline$-11-69$ & $30: 195$ & $-2.80 \pm 0.65$ & $-25.1 \pm 6.5$ a & & \\
\hline$-11-81$ & $30: 195$ & $-1.81 \pm 0.52$ & $-15.1 \pm 5.2 \mathrm{a}$ & & \\
\hline$-11-198$ & $30: 195$ & $-0.26 \pm 0.69$ & $+0.4 \pm 6.9$ a & & \\
\hline$-12-47$ & $30: 195$ & $+0.73 \pm 0.35$ & $+10.3 \pm 3.5 \mathrm{a}$ & & \\
\hline$-12-61$ & $30: 195$ & $+0.91 \pm 0.35$ & $+12.1 \pm 3.5 \mathrm{a}$ & & \\
\hline$-12-80$ & $30: 195$ & $+0.13 \pm 0.35$ & $+4.3 \pm 3.5 \mathrm{a}$ & & \\
\hline$-18-11$ & $30: 197$ & $11,930 \pm 290$ & & & 9980 B.C. \\
\hline$-21-18$ & $30: 197$ & $11,550 \pm 280$ & & & 9600 B.C. \\
\hline$-23-22$ & $30: 197$ & $9200 \pm 300$ & & & 7250 B.C. \\
\hline$-26-23$ & $30: 197$ & $9030 \pm 350$ & & & 7080 B.C. \\
\hline$-27-25$ & $30: 197$ & $3720 \pm 150$ & & & 1770 B.C. \\
\hline$-28-33$ & $30: 197$ & $3970 \pm 170$ & & & 2020 B.C. \\
\hline$-29-146$ & $30: 197$ & $5140 \pm 115$ & & & 3190 B.C. \\
\hline$-30-145$ & $30: 197$ & $5020 \pm 105$ & & & 3070 B.C. \\
\hline$-31-67$ & $30: 196$ & $13,050 \pm 270$ & & & 11,100 B.C. \\
\hline$-32-60$ & $30: 196$ & $15,700 \pm 350$ & & & 13,750 B.C. \\
\hline$-32-118 a$ & $30: 196$ & $17,100 \pm 560$ & & & 15,150 B.C. \\
\hline$-32-118 c$ & $30: 196$ & $12.850 \pm 500$ & & & 10,900 B.C. \\
\hline$-38-121 A$ & $30: 196$ & $12,000 \pm 200$ & & & 10,050 B.C. \\
\hline$-38-121 B$ & $30: 196$ & $12,300 \pm 300$ & & & 10,350 B.C. \\
\hline$-38-121 C$ & $30: 196$ & $6150 \pm 500$ & & & 4200 B.C. \\
\hline$-40-34$ & $30: 197$ & $3120 \pm 160$ & & & 1170 B.C. \\
\hline$-54-44 / 54$ & $30: 197$ & $2060 \pm 90$ & & & 110 B.C. \\
\hline$-55-50$ & $30: 195$ & $+1.29 \pm 0.43$ & $+15.9 \pm 4.3 \mathrm{a}$ & & \\
\hline$-55-147$ & $30: 195$ & $-0.34 \pm 0.41$ & $-0.4 \pm 4.1 \mathrm{a}$ & & \\
\hline$-59-57$ & $30: 197$ & $2010 \pm 60$ & & & 60 B.C. \\
\hline$-61-149$ & $30: 197$ & $5140 \pm 130$ & & & 3190 B.C. \\
\hline$-63-36$ & $30: 197$ & $508 \pm 85$ & & & A.D. 1442 \\
\hline$-67-49$ & $30: 198$ & $2010 \pm 80$ & & & 60 B.C. \\
\hline$-70-102$ & $30: 198$ & $1390 \div 120$ & & & A.D. 560 \\
\hline$-71-85$ & $30: 198$ & $2100 \pm 100$ & & & $150 \mathrm{B.C.}$ \\
\hline$-72-88$ & $30: 198$ & $2050 \pm 110$ & & & 100 B.C. \\
\hline$-74-52$ & $30: 195$ & $-3.15 \pm 0.6$ & $-28.6 \pm 6.0 \mathrm{a}$ & & \\
\hline$-75-68$ & $30: 197$ & $11,450 \pm 180$ & & & 9500 B.C. \\
\hline$-77-54$ & $30: 196$ & $12,300 \pm 260$ & & & 10,350 B.C. \\
\hline$-78-58$ & $30: 195$ & $-2.63 \pm 0.52$ & $-23.4 \pm 5.2 \mathrm{a}$ & & \\
\hline$-81-62$ & $30: 199$ & $3040 \pm 100$ & & & 1090 B.C. \\
\hline$-85-91$ & $30: 196$ & $11,300 \pm 220$ & & & 9350 B.C. \\
\hline$-87-76$ & $30: 197$ & $1070 \pm 80$ & & & A.D. 880 \\
\hline$-88-74$ & $30: 196$ & $13,250 \pm 280$ & & & 11,300 B.C. \\
\hline$-89-70$ & $30: 198$ & $2040 \pm 50$ & & & 90 B.C. \\
\hline$-89-70 a$ & $30: 198$ & $2150 \pm 75$ & & & 200 B.C. \\
\hline$-89-135$ & $30: 198$ & $2000 \pm 65$ & & & 50 B.C. \\
\hline $.91-71$ & $30: 197$ & $2240 \pm 70$ & & & 290 B.C. \\
\hline$-91-126 a$ & $30: 197$ & $1885 \pm 80$ & & & A.D. 65 \\
\hline$-91-126 b$ & $30: 197$ & $1260 \pm 150$ & & & A.D. 690 \\
\hline-93.73 & $30: 197$ & $1905 \pm 65$ & & & A.D. 45 \\
\hline $.94-72$ & $30: 197$ & $2060 \pm 110$ & & & 110 B.C. \\
\hline$-100-101$ & $30: 198$ & $1930 \pm 100$ & & & A.D. 20 \\
\hline$-101-115$ & $30: 198$ & $555 \pm 90$ & & & A.D. 1395 \\
\hline$-102-137$ & $30: 199$ & $2000 \pm 60$ & & & 50 B.C. \\
\hline$-104-90$ & $30: 199$ & $3590 \pm 130$ & & & 1640 B.C. \\
\hline$-105-87$ & $30: 197$ & $11,500 \pm 300$ & & & 9550 B.C. \\
\hline$-106-89$ & $30: 196$ & $12.700 \pm 320$ & & & 10,750 B.C. \\
\hline$-119-103$ & $30: 198$ & $4040 \pm 150$ & & & 2090 B.C. \\
\hline
\end{tabular}




\begin{tabular}{|c|c|c|c|c|}
\hline $\begin{array}{l}\text { Laboratory } \\
\text { number }\end{array}$ & $\begin{array}{l}\text { Published } \\
\text { reference }\end{array}$ & $\begin{array}{l}\text { Original date or } \\
\text { other value }\end{array}$ & $\begin{array}{l}\text { Corrected date } \\
\text { or other value }\end{array}$ & $\partial \mathrm{C}^{14}, \%$ \\
\hline$-122-100$ & $30: 199$ & $5860 \pm 150$ & & \\
\hline$-124-138$ & $30: 199$ & $1850 \pm 75$ & & \\
\hline$-125-107$ & $30: 197$ & $5200 \pm 200$ & & \\
\hline$-126-143$ & $30: 197$ & $9290 \pm 190$ & & \\
\hline$-129-104$ & $30: 196$ & $10,200 \pm 250$ & & \\
\hline$-130-114$ & $30: 196$ & $10,150 \pm 230$ & & \\
\hline$-135-111$ & $30: 195$ & $-2.1 \pm 0.65$ & $-18.0 \pm 6.5 \mathrm{a}$ & \\
\hline$-136-116$ & $30: 196$ & $12,980 \pm 370$ & & \\
\hline$-138-123$ & $30: 198$ & $6070 \pm 160$ & & \\
\hline$-139-129$ & $30: 198$ & $2200 \pm 90$ & & \\
\hline$-141-120 / 166$ & $\begin{array}{ll}6 & 30: 198\end{array}$ & $3825 \pm 85$ & & \\
\hline$-142-133$ & $30: 198$ & $2465 \pm 170$ & & \\
\hline$-145-117$ & $30: 199$ & $7480 \pm 200$ & & \\
\hline$-145-127$ & $30: 199$ & $3370 \pm 90$ & & \\
\hline$-146-124$ & $30: 199$ & $3700 \pm 120$ & & \\
\hline$-148-128$ & $30: 198$ & $2540 \pm 100$ & & \\
\hline$-149-132$ & $30: 198$ & $2690 \pm 75$ & & \\
\hline$-150-139$ & $30: 198$ & $2365 \pm 60$ & & \\
\hline$-150-148$ & $30: 198$ & $2460 \pm 75$ & & \\
\hline$-152-199$ & $30: 195$ & $+0.47 \pm 0.78$ & $+7.7 \pm 7.8 \mathrm{a}$ & \\
\hline$-155-189$ & $30: 195$ & $-0.26 \pm 0.69$ & $+0.4 \pm 6.9 \mathrm{a}$ & \\
\hline$-160-187$ & $30: 195$ & $-2.80 \pm 0.9$ & $-25.1 \pm 9.0 \mathrm{a}$ & \\
\hline $.161-177$ & $30: 195$ & $-3.97 \pm 0.82$ & $-36.8 \pm 8.2 \mathrm{a}$ & \\
\hline$-162-162$ & $30: 195$ & $-3.23 \pm 0.73$ & $-29.4 \pm 7.3 \mathrm{a}$ & \\
\hline $.163-156$ & $30: 198$ & $1240 \pm 60$ & & \\
\hline$-164-160$ & $30: 198$ & $1500 \pm 80$ & & \\
\hline $.165-157$ & $30: 198$ & $2920 \pm 65$ & & \\
\hline $.166-158$ & $30: 197$ & $1915 \pm 65$ & & \\
\hline$-167-159$ & $30: 198$ & $1840 \pm 75$ & & \\
\hline$-168-161$ & $30: 199$ & $2950 \pm 75$ & & \\
\hline $.169-210$ & $30: 197$ & $1930 \pm 80$ & & \\
\hline$-171-163$ & $30: 198$ & $2100 \pm 65$ & & \\
\hline$-179-171$ & $30: 195$ & $-2.1 \pm 0.75$ & $-18.0 \pm 7.5 \mathrm{a}$ & \\
\hline$-180-173$ & $30: 198$ & $2560 \pm 100$ & & \\
\hline$-181-181$ & $30: 198$ & $2755 \pm 100$ & & \\
\hline$-182-203$ & $30: 198$ & $2050 \pm 75$ & & \\
\hline$-183-217$ & $30: 198$ & $1965 \pm 65$ & & \\
\hline$-184-202$ & $30: 198$ & $2915 \pm 85$ & & \\
\hline$-193-178$ & $30: 196$ & $4330 \pm 360$ & & \\
\hline$-230-235$ & $30: 198$ & $1360 \pm 60$ & & \\
\hline$-231-204$ & $30: 198$ & $1265 \pm 55$ & & \\
\hline$-232-211$ & $30: 198$ & $1185 \pm 70$ & & \\
\hline
\end{tabular}

A.D./ B.C. date 3910 B.C. A.D. 100 3250 B.C. 7340 B.C. 8250 B.C. 8200 B.C.

11,030 B.C. 4120 B.C. 250 B.C.

1875 B.C.

515 B.C.

5530 B.C.

1420 B.C.

1750 B.C.

590 B.C.

740 B.C.

415 B.C.

510 B.C.
A.D. 710
A.D. 450
970 B.C.
A.D. 35
A.D. 110
1000 B.C.
A.D. 20
150 B.C.
610 B.C.
805 B.C.
100 B.C.
15 B.C.
965 B.C.
2380 B.C.
A.D. 590
A.D. 685

A.D. 765

A.D./

B.C. date

10,050 B.C. 1520 B.C. 8270 B.C. 6920 B.C. 7500 B.C. 7400 R.C. 6470 B.C. 7490 B.C.

8440 B.C.

10,675 B.C. 5700 B.C.

11,375 B.C. 